\title{
Electron-ion collision rates in noble gas clusters irradiated by femtosecond laser pulse
}

\author{
R. Dey ${ }^{1,}$ a and A. C. Roy ${ }^{2}$ \\ ${ }^{1}$ Max-Planck-Institut für Plasmaphysik, EURATOM Association, \\ Boltzmannstr. 2, 85748 Garching, Germany \\ ${ }^{2}$ School of Mathematical Sciences, Ramakrishna Mission Vivekananda University, \\ Belur Math 711202, West Bengal, India
}

\begin{abstract}
We report a theoretical analysis of electron-ion collision rates in xenon gas clusters irradiated by femtosecond laser pulses. The present analysis is based on the eikonal approximation (EA), the first Born approximation (FBA) and the classical (CL) methods. The calculations are performed using the plasma-screened Rogers potential introduced by Moll et al. (M. Moll, P. Hilse, M. Schlanges, Th. Bornath, V. P. Krainov, J. Phys. B. 43, 135103 (2010)) as well as the Debye potential for a wide range of experimental parameters. We find that the magnitudes of electron-ion collision frequency obtained in the EA do not fall as rapidly with the kinetic energy of electrons as in the FBA and CL methods for higher charge states of xenon ion $\left(\mathrm{Xe}^{8+}\right.$ and $\left.\mathrm{Xe}^{14+}\right)$. Furthermore, EA shows that the effect of the inner structure of ion is most dominant for the lowest charge state of xenon ion $\left(\mathrm{Xe}^{1+}\right)$. In the case of the present effective potential, FBA overestimates the CL results for all three different charge states of xenon, whereas for the Debye potential, both the FBA and CL methods predict collision frequencies which are nearly close to each other.
\end{abstract}

\footnotetext{
a e-mail: ritud@ipp.mpg.de
} 


\section{INTRODUCTION}

The high density and high temperature plasmas can be produced by the interactions of atomic clusters with intense ultrashort laser pulses. In recent years, a number of ex-

periments have been carried out in laboratory plasmas [1-6]. On the other hand, various theoretical attempts have been made to study the laser-cluster interactions process [3, 7-13]. The physical processes which occur in the clusters irradiated by the laser fields are (i) photoionization of atoms, (ii) inverse bremsstrahlung heating, (iii) inelastic and elastic collisions between atoms/ions and electrons, (iv) many-body recombination of ions with electrons and (v) the electromagnetic interaction of electrons/ions with the laser field. The screening due to plasma environment and proper choice of atomic potential play important roles for calculating transport cross section, collision frequency, inverse bremsstrahlung cross section etc. when the above physical processes are concerned. Recently, inverse bremsstrahlung (IB) cross sections estimated within the evolving plasmas are studied by Wang et al. [14]. They applied the first Born approximation (FBA) method to evaluate IB cross sections in both the weakly and strongly coupled plasmas. Their results show that the above cross section estimated using the effective atomic potentials is not affected much by the plasma environment. Their observation validates the estimations of the enhanced heating effect obtained by Walters et al. [10]. Very recently, Moll et al. [15] calculated electron-ion collision rates in atomic clusters irradiated by femtosecond laser pulses and found that FBA was not applicable for the wide range of experimental parameters. They considered the energy region, $\mathrm{E}<2 Z_{i}^{2}$, where $\mathrm{E}$ represents the mean kinetic energy (in a.u.) of the quasi-free electrons inside the cluster, and $\mathrm{Z}_{i}$ stands for the ion charge number. In essence, this is the energy region where classical methods should be valid. They also calculated electron-ion collision frequency for argon, krypton and xenon ions using a classical method and pointed out that it was important to take into account the inner sturucture of ions and the screening by the surrounding plasma medium.

In the present paper, we have concentrated on the ponderomotive heating of the cluster. This heating is due to the acceleration of the electrons in the presence of multiply charged atomic ions inside the cluster. The screening due to the plasma electrons can be accounted through the Debye potential. We have applied the eikonal approximation (EA) $[16,17]$ to calculate the electron-ion collision frequency using the Debye potential as well as a realistic 
model potential given by Rogers [18] in the applicable experimental regime [19]. Besides EA, we have also examined the effectiveness of FBA and classical (CL) methods in order to predict the electron-ion collision frequency which determines the absorption of laser energy in the plasma. In contrast to the FBA, EA contains higher-order contributions. The eikonal amplitude also satisfies the optical theorem within its range of validity [20]. In the present study, besides the classical region, $\mathrm{E}<2 Z_{i}^{2}$, we have also extended our investigation in the energy region $\mathrm{E}>2 Z_{i}^{2}$, where the perturbative methods are valid. A comparison is made between the predictions of EA with the corresponding results obtained in the FBA and CL methods throughout the two energy regions. It is worth stressing that the EA has been found to be successful in a wide variety of collisions [21-27] such as ionization, ionization-excitation etc. .

\section{THEORY}

\subsection{Effective atomic potential}

In the present investigation we have used the following atomic potential as was introduced by Rogers [18] to calculate electron-ion collision cross sections, (atomic units are used throughout, unless otherwise indicated),

$$
V(r)=-\frac{1}{r}\left(Z_{i}+\sum_{n=1}^{n^{*}} N_{n} e^{-\alpha_{n} r}\right)
$$

where $N_{n}$ is the number of electrons in the $n$th occupied electron shell and $n^{*}$ is the number of shells. The screening parameters $\alpha_{n}$ are taken from [18]. In Eq.(1) $Z_{i}$ is the ion charge number. Normally the ions and electrons inside the plasma interact via an electrostatic interaction potential. A simple form of this potential is the Debye potential and is given by

$$
V_{D}(r)=-\frac{Z_{i}}{r} \exp \left(-\kappa_{D} r\right)
$$

where $\kappa_{D}$ is the inverse of the plasma Debye screening length $\lambda_{D}$,

$$
\lambda_{D}=\left[\frac{k_{B} T_{e}}{4 \pi n_{i} \bar{Z}_{i}}\right]^{1 / 2} .
$$


Here, $k_{B}$ is the Boltzmann constant and $n_{i}$ represents the number density of atomic ions inside the cluster. For simplicity, the mean ion charge $\bar{Z}_{i}$ is replaced by the respective ion charge $Z_{i}$. $T_{e}$ is the electron temperature which is related to the mean kinetic energy of electron as follows:

$$
E=\frac{3 k_{B} T_{e}}{2}
$$

In order to account for the surrounding plasma medium an additional Debye screening factor is introduced in Eqn(1) and then the resultant expression for the potential becomes

$$
V_{s c}(r)=-\frac{1}{r}\left(Z_{i}+\sum_{n=1}^{n^{*}} N_{n} e^{-\alpha_{n} r}\right) \exp \left(-\kappa_{D} r\right)
$$

Next, we will describe two different approches, quantum mechanical and classical, to calculate the transport cross section for the electron-ion collisions.

\subsection{Quantum mechanical calculations}

For spherically symmetric potentials, the eikonal scattering amplitude for electron-atom collision [17] is given by

$$
F(\theta)=\frac{k}{i} \int_{0}^{\infty} d b J_{0}(q b)[\exp [i(\chi(b))]-1]
$$

where

$$
\chi(b)=-\frac{1}{k} \int_{-\infty}^{\infty} d z V(b, z)
$$

and

$$
q=2 k \sin \left(\frac{\theta}{2}\right) .
$$

Here, $\mathrm{k}$ is the wavenumber of the incident particle, $\mathrm{J}_{0}$ is the zeroth-order Bessel function of the first kind, $\mathrm{q}$ is the magnitude of momentum transfer and $\mathrm{V}$ is the atomic potential. $\mathrm{b}$ and $\mathrm{z}$ denote the two components of the position vector $\mathbf{r}$, and are defined as $\mathbf{r}=\mathbf{b}+\hat{\mathbf{k}} z$. The impact parameter $\mathrm{b}$ is perpendicular to the incident $\mathrm{z}$ direction. 
The FBA amplitude is calculated in the same way as in Moll et al.[15]. The quantum momentum transport cross section is defined by

$$
\sigma_{t r}=\int d \Omega \frac{d \sigma}{d \Omega}(1-\cos (\theta))
$$

where $\theta$ and $\Omega$ are the scattering angle and the solid angle, respectively. The differential cross section can be obtained from Eqn(4) and is written as,

$$
\frac{d \sigma}{d \Omega}=|F(\theta)|^{2}
$$

\subsection{Classical calculation}

The momentum transport cross section $\left(\sigma_{t r}\right)$ for classical elastic scattering is given by

$$
\sigma_{t r}=2 \pi \int_{0}^{b_{\max }} d b b\left(1-\cos \theta_{C L}(b)\right)
$$

where the scattering angle $\theta_{C L}$ is related to the deflection angle $\Theta(b)$ [28] such as

$$
\Theta(b)= \pm \theta_{C L}(b)-2 \pi n, \quad \theta_{C L} \in[0, \pi] .
$$

The angle $\Theta(b)$ can also be expressed in terms of $V(r)$

$$
\Theta(b)=\pi-2 \int_{r_{\min }}^{\infty} d r r^{-2}\left[b^{-2}\left(1-\frac{V(r)}{E}\right)-r^{-2}\right]^{-1 / 2}
$$

Here $r_{\min }$ is the classical distance of closest approach. The maximum value of $b\left(b_{\max }\right)$ is taken to be $\mathrm{b}_{\max }=\mathrm{n}_{i}^{-1 / 3}$ which was also used by Moll et al. [15].

We have evaluated the one-dimensional integrals (in case of both the FBA and EA methods) numerically to calculate momentum transport cross sections for electron-ion scattering. For the estimation of classical elastic scattering angle $\left(\theta_{C L}\right)$, we have followed the same procedure as was proposed by Neumann et al. [29].

The electron-ion collision frequency $\nu_{e i}$ is given by

$$
\nu_{e i}=n_{i}\left\langle v \sigma_{t r}(v)\right\rangle \approx n_{i} v_{e} \sigma_{t r}\left(v_{e}\right)
$$



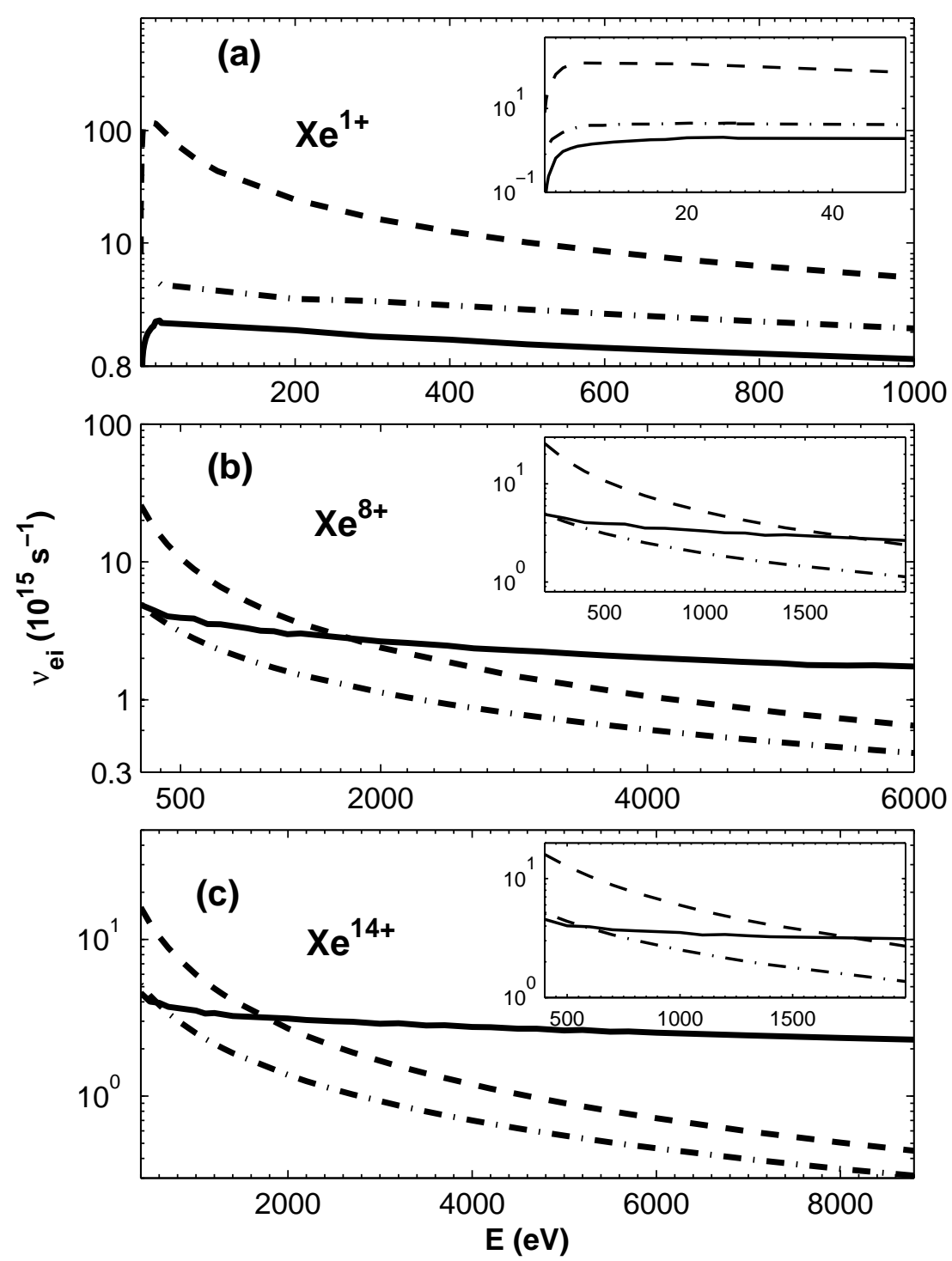

FIG. 1: Electron-ion collision frequency calculated with the plasma-screened Rogers potential as a function of kinetic energy of electrons for a fixed value of $n_{i}=0.00239$ a.u. for (a) $\mathrm{Xe}^{1+}$ (b) $\mathrm{Xe}^{8+}$ and (c) $\mathrm{Xe}^{14+}$ ions. The solid curve represents the present EA results. The dashed curve is the present FBA results. The dashed-dot curve represents the present classical results. The inset represents the extended view of the classical region.

where the mean electron velocity is calculated from

$$
v_{e}=\sqrt{2 E}=\sqrt{3 k_{B} T_{e}}
$$

Here the validity condition is that the laser frequency has to be greater than the electron-ion collision frequency, the same as was mentioned by Moll et al. [15]. 

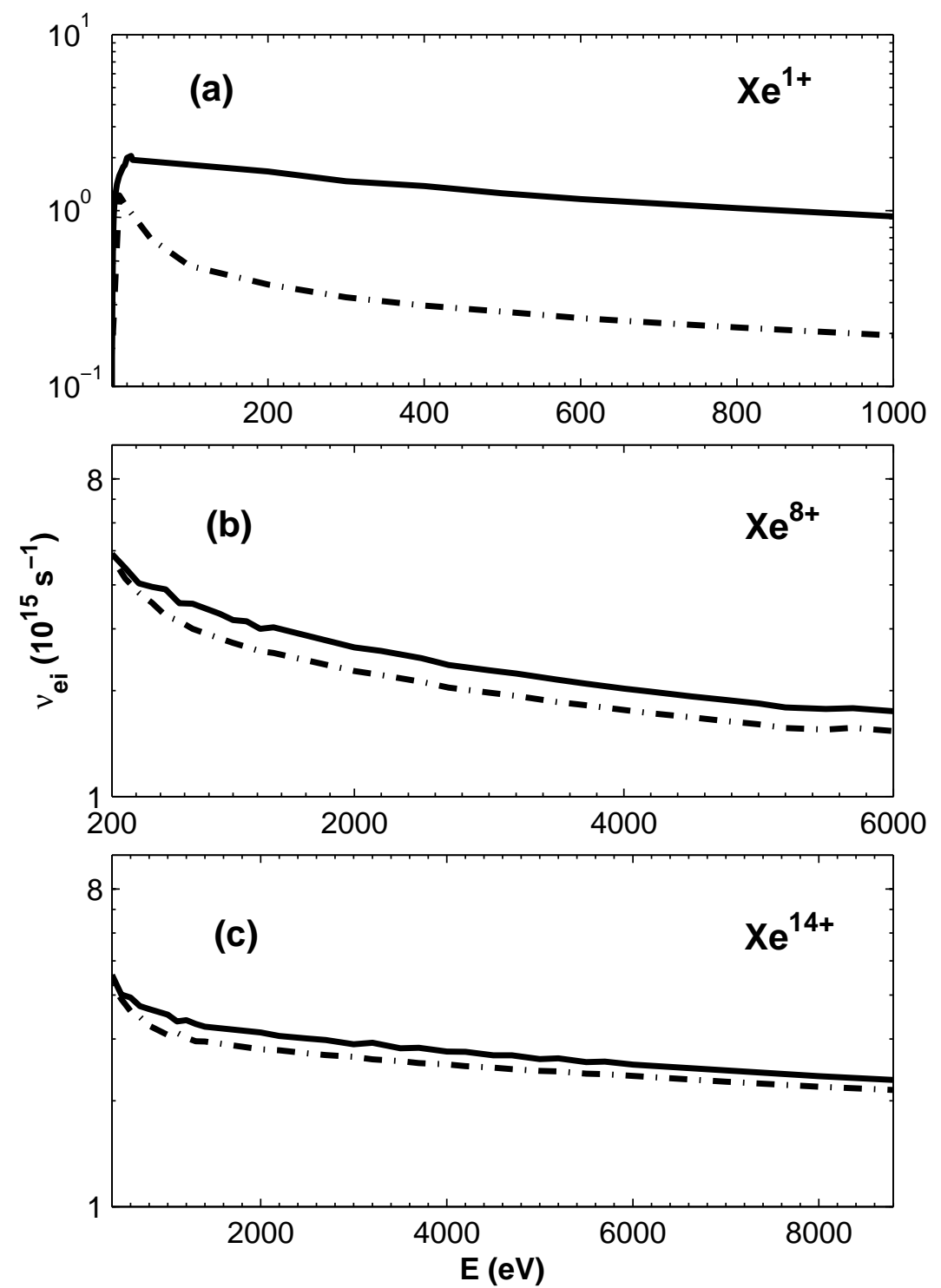

FIG. 2: Electron-ion collision frequency as a function of kinetic energy of electrons for a fixed value of $n_{i}=0.00239$ a.u. for (a) $\mathrm{Xe}^{1+}$ (b) $\mathrm{Xe}^{8+}$ and (c) $\mathrm{Xe}^{14+}$ ions. The solid curve represents the present EA results for the plasma-screened Rogers potential. The dashed-dot curve is the present EA results with the Debye potential.

\section{RESULTS AND DISCUSSION}

Fig.1 shows the present electron-ion collision frequency obtained in the EA, FBA and CL methods as a function of kinetic energy E for the plasma-screened effective potential $V_{s c}$ for $\mathrm{Xe}^{1+}, \mathrm{Xe}^{8+}$ and $\mathrm{Xe}^{14+}$ ions. In view of the estimation of the mean electron energies of the charged Xe ions by Petrov and Davis [19] we have limited the energy region upto 1, 6 and $8.8 \mathrm{KeV}$ for $\mathrm{Xe}^{1+}, \mathrm{Xe}^{8+}$ and $\mathrm{Xe}^{14+}$ ions, respectively. We have considered the ion number 

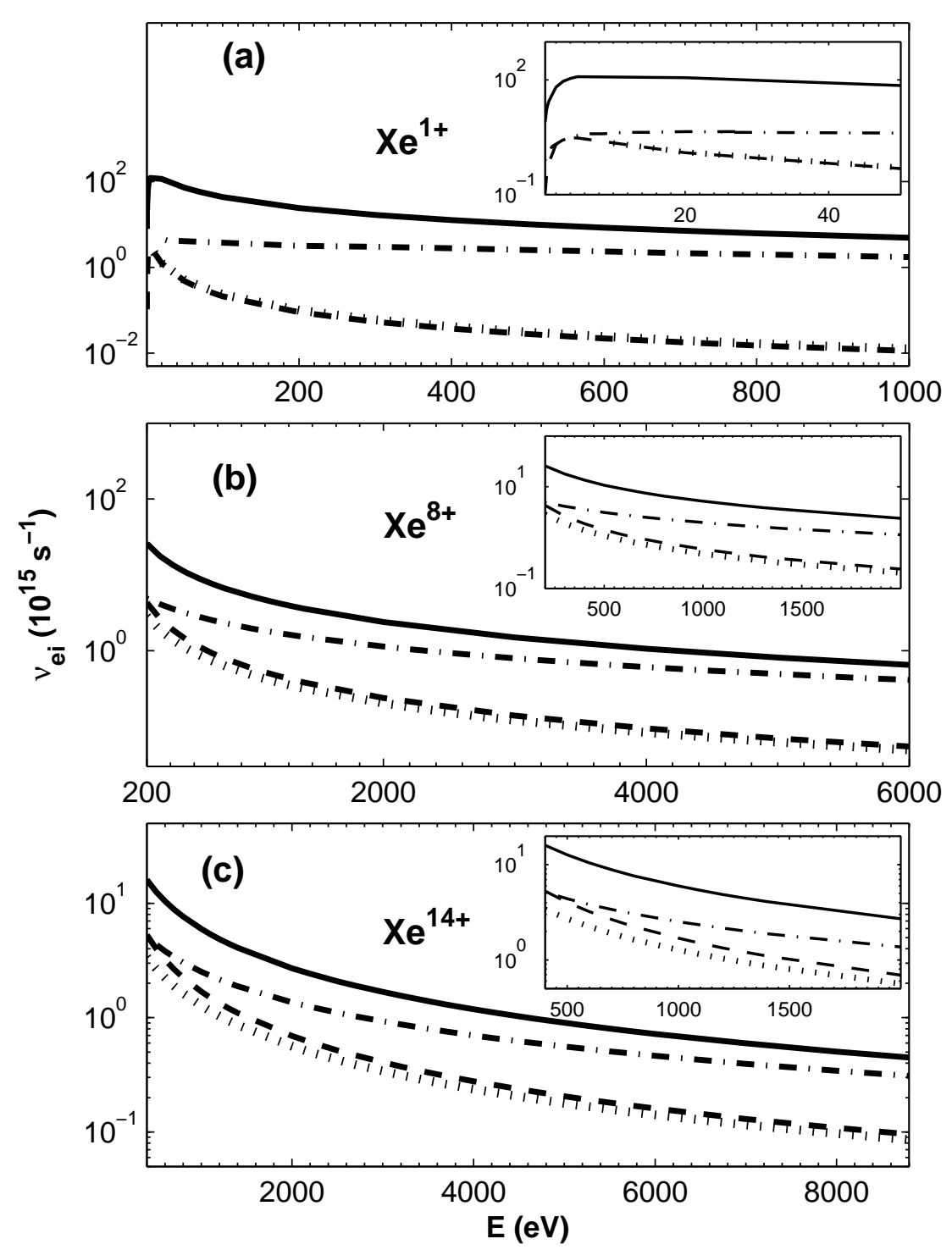

FIG. 3: Electron-ion collision frequency as a function of kinetic energy of electrons for a fixed value of $n_{i}=0.00239$ a.u. for (a) $\mathrm{Xe}^{1+}$ (b) $\mathrm{Xe}^{8+}$ and (c) $\mathrm{Xe}^{14+}$ ions. The solid curve represents the present FBA results with the potential (3). The dashed curve displays the present FBA results with the potential (2). The dashed-dot curve represents the present CL results with the potential (3). The dotted curve is the present CL results with the potential (2). The inset represents the extended view of the classical region.

density 0.00239 a.u. for xenon which is the same as was used by Moll et al. [15]. In the present calculation, the electron density $n_{e}$ for the quasi-free electrons inside the plasma is taken to be $n_{e}=n_{i} Z_{i}$. Consequently, the electron densities for $\mathrm{Xe}^{1+}, \mathrm{Xe}^{8+}$ and $\mathrm{Xe}^{14+}$ ions are $1.610 \times 10^{22}, 1.290 \times 10^{23}$ and $2.263 \times 10^{23} \mathrm{~cm}^{-3}$, respectively. The screening parameter $\left(\kappa_{D}\right)$ varies from $0.066-3 \AA^{-1}, 0.075-0.417 \AA^{-1}$ and $0.083-0.550 \AA^{-1}$ for $\mathrm{Xe}^{1+}, \mathrm{Xe}^{8+}$ and $\mathrm{Xe}^{14+}$ ions, respectively, for the energy range considered here. We notice that the $\nu_{e i}$ predicted by 
FBA and CL methods falls rapidly with the increase of kinetic energy of electrons for $\mathrm{Xe}^{8+}$ and $\mathrm{Xe}^{14+}$ ions. We also observe that FBA frequencies are much higher than those of the EA and classical calculations throughout the energy range considered here for the lowest charge state of xenon ion $\left(\mathrm{Xe}^{1+}\right)$. Furthermore, the CL results overestimate the corresponding EA values by a factor of about 2 in the whole energy range for $\mathrm{Xe}^{1+}$ ion (see, the inset of panel (a) for the classical energy region). For the $\mathrm{Xe}^{8+}$ and $\mathrm{Xe}^{14+}$ ions, FBA yields frequencies higher than the EA and CL methods upto the electron energy E $\approx 2 \mathrm{KeV}$ (see, the inset of panel (b) and (c) of Fig.1). Beyond this, FBA results overestimate CL calculation by about 1.57-2.10 (1.44-1.98) times and underestimate the EA by 1.10-2.68 (1.15-5.12) times for $\mathrm{Xe}^{8+}$ $\left(\mathrm{Xe}^{14+}\right)$ ion in the entire energy range. It is worth noting that the qualitative nature of FBA, EA and CL curves is almost similar for both the higher charge states of xenon ion, although the magnitudes differ by a significant margin. For example, the EA magnitude deviate by about $30 \%$ in the energy range $2-8.8 \mathrm{KeV}$ while FBA and classical results show variations of $83 \%$ and $77 \%$, respectively for $\mathrm{Xe}^{14+}$ ion. EA is expected to yield reliable results at these higher energies (for example, $\mathrm{E}>54.4 \mathrm{eV}$ for $\mathrm{Xe}^{1+}$ and $\mathrm{E}>3.7 \mathrm{KeV}$ for $\mathrm{Xe}^{8+}$ ) because this energy range is nearly appropriate for the applicability of eikonal approximation. At higher energies, FBA is seen to fall steeper than the EA (see panel (c) of Fig.1). The reason for the discrepancy may be ascribed to the fact that FBA is not applicable for strong potential. On the other hand, the CL method is also not quite expected to be valid for these high energy regimes, especially at $\mathrm{E}>54.4 \mathrm{eV}$ for $\mathrm{Xe}^{1+}$ and $\mathrm{E}>3.7 \mathrm{KeV}$ for $\mathrm{Xe}^{8+}$.

Fig. 2 exhibits a comparison of the eikonal collision frequencies for the screened Rogers potential $V_{s c}$ with the corresponding results for the Debye potential $V_{D}$ in the case of $\mathrm{Xe}^{1+}$, $\mathrm{Xe}^{8+}$ and $\mathrm{Xe}^{14+}$ ions. For higher charge states of xenon ion $\left(\mathrm{Xe}^{8+}\right.$ and $\left.\mathrm{Xe}^{14+}\right)$, eikonal results are not very sensitive to the choice of potentials. However, the collision frequencies calculated with the screened Rogers potential are slightly greater than those with the Debye potential in the present range of kinetic energy of electrons. In the case of the lowest charge state of xenon ion $\left(\mathrm{Xe}^{1+}\right)$, EA frequencies computed with the Debye potential are found to be much lower than the corresponding EA results obtained with the screened Rogers potential. Evidently, for the lowest charge state and for the lower kinetic energy of electrons the inner structure of the ions is responsible for the big difference between the two results.

Fig. 3 displays the present FBA and CL results for the two different potentials $V_{s c}$ and $V_{D}$. We notice that the collision frequencies obtained with the screened Rogers potential 
$V_{s c}$ are much higher than those computed with the Debye potential $V_{D}$ for both the FBA and CL methods. This clearly reflects the influence of the inner structure of the ions. On the other hand, in the case of Debye potential both the FBA and CL methods predict collision frequencies which nearly coincide with each other. Furthermore, for $\mathrm{Xe}^{1+}$ ion, collision frequencies predicted by the CL method for the screened Rogers potential are almost constant in the classical region $(\mathrm{E}>5 \mathrm{eV})$ while those computed using the above method for the Debye potential fall rapidly along E (see, inset of panel (a) of Fig.3).

\section{CONCLUSIONS}

Electron-ion collisions are investigated for plasma conditions in a xenon gas cluster where the ponderomotive heating of the cluster is considered. The present calculations for the electron-ion collision frequency are performed using the EA, FBA and CL methods for the plasma-screened Rogers potential as well as the Debye potential for three different charge states of xenon $\left(\mathrm{Xe}^{1+}, \mathrm{Xe}^{8+}\right.$ and $\left.\mathrm{Xe}^{14+}\right)$. In a wide range of experimental parameters, the magnitudes of EA do not fall as rapidly with the electron energy as those obtained in the FBA and CL methods for higher charge states of xenon ion $\left(\mathrm{Xe}^{8+}\right.$ and $\left.\mathrm{Xe}^{14+}\right)$. EA also shows that the effect of the inner structure of ion is most dominant in the lowest charge state of xenon ion $\left(\mathrm{Xe}^{1+}\right)$. It does not depend very much on the choice of potentials. In the case of plasma-screened Rogers potential, FBA is found to overestimate the CL results for all three different charge states of xenon ion. However, for the Debye potential, both FBA and CL methods predict collision frequencies which are nearly close to each other.

\section{ACKNOWLEDGMENT}

One of the authors (R. D.) is grateful to Professor Arne Kallenbach for his kind help.

[1] H. Wabnitz, L. Bittner, A. R. B. de Castro, R. Döhrmann, P. Gürtler, T. Laarmann, W. Laasch, J. Schulz, A. Swiderski, K. von Haeften, T. Möller, B. Faatz, A. Fateev, J. Feldhaus, C. Gerth, U. Hahn, E. Saldin, E. Schneidmiller, K. Sytchev, K. Tiedtke, R. Treusch, M. Yurkovk, Nature (London) 420, 482 (2002). 
[2] T. Laarmann, M. Rusek, H. Wabnitz, J. Schulz, A. R. B. de Castro, P. Gürtler, W. Laasch, T. Möller, Phys. Rev. Lett. 95, 063402 (2005).

[3] U. Saalmann, Ch. Siedschlag, J. M. Rost, J. Phys. B 39, R 39 (2006).

[4] C. Deiss, N. Rohringer, J. Burgdörfer, E. Lamour, C. Prigent, J. P. Rozet, D. Vernhet, Phys. Rev. Lett. 96, 013203 (2006).

[5] J. Jha, M. Krishnamurthy, J. Phys. B: At. Mol. Opt. Phys. 41, 041002 (2008).

[6] H. Thomas, C. Bostedt, M. Hoener, E. Eremina, H. Wabnitz, T. Laarmann, E. Plönjes, R. Treusch, A. R. B. de Castro, T. Möller, J. Phys. B: At. Mol. Opt. Phys. 42, 134018 (2009).

[7] N. M. Kroll, K. M. Watson, Phys. Rev. A 8, 804 (1973).

[8] V.P. Krainov, M.B. Smirnov, Phys. Rep. 370, 237 (2002).

[9] R. Santra, C. H. Greene, Phys. Rev. Lett. 91, 233401 (2003).

[10] Z. B. Walters, R. Santra, C. H. Greene, Phys. Rev. A. 74, 043204 (2006).

[11] P. Mulser, R. Schneider, J. Phys. A: Math. Theor. 42, 214058 (2009).

[12] T. Fennel, K.-H. Meiwes-Broer, J. Tiggesbäumker, P.-G. Reinhard, P. M. Dinh, E. Suraud, Rev. Mod. Phys. 82, 1793 (2010).

[13] M. Arbeiter, T. Fennel, New Journal of Physics 13, 053022 (2011).

[14] F. Wang, E. Weckert, B. Ziaja, J. Plasma Physics 75, 289 (2009).

[15] M. Moll, P. Hilse, M. Schlanges, Th. Bornath, V. P. Krainov, J. Phys. B. 43, 135103 (2010).

[16] R. J. Glauber, in: W. E. Brittin, et al (Eds), Lectures in the Theoretical Physics, Vol. I, (Interscience New Work), p.315 (1959).

[17] A. C. Roy, N. C. Sil, J. Phys. B: Atom Molec. Phys. 11, 2729 (1978).

[18] F. J. Rogers, Phys. Rev. A 23, 1008 (1981).

[19] G. M. Petrov, J. Davis, Phys. of Plasmas 15, 056705 (2008).

[20] E. Gerjuoy, B.K. Thomas, Rep. Prog. Phys. 37, 1345 (1974).

[21] R. Dey, A. C. Roy, Phys. Lett. A 332, 60 (2004).

[22] R. Dey, A. C. Roy, Phys. Lett. A 353, 341 (2006).

[23] R. Dey, A. C. Roy , C. Dal Cappello, Nucl. Instr. Methods B 266, 242 (2008).

[24] R. Dey, A. C. Roy, Nucl. Instr. Methods B 267, 2357 (2009).

[25] M. Schulz, A. C. Laforge, K. N. Egodapitiya, J. S. Alexander, A. Hasan, M. F. Ciappina, A. C. Roy, R. Dey, A. Samolov, A. L. Godunov, Phys. Rev. A 81, 052705 (2010) .

[26] R. Dey, A. C. Roy, Nucl. Instr. Methods B 269, 364 (2011). 
[27] R. Dey, A. C. Roy, C. Dal. Cappello, Nucl. Instr. Methods B 271, 82 (2012).

[28] R. G. Newton, Scattering Theory of Waves and Particles (Berlin: Springer), (1982).

[29] H. Neumann, T. Q. Le, B. Van Zyl, Phys. Rev. A 15, 1887 (1977). 